\title{
Research on application of Hilbert transform in radar signal simulation
}

\author{
Longjin Zheng, Zhixin Liu, Guangtao Wang, Zhaoqiao Zhang \\ School of Resource and Geoscience \\ CUMT \\ Xuzhou \\ Lonjzheng@163.com
}

\begin{abstract}
At present, the radar is used to analyze the radar time range profile, which is mainly based on the kinematic characteristics of radar wave. With the constantly mature of the analytic method of signal analysis of GPR, we can effectively extract the "three instantaneous attributes" (the instantaneous amplitude, instantaneous phase and instantaneous frequency) from complex signal, using the analytic signal composed by original signal and the virtual signal. The virtual signal transformed from original radar signal by Hilbert transform. Basing on the analysis of previous research status, the author makes a detailed study on the excellent performance of the GPR signal processing by using Hilbert transform in this method. The instantaneous parameter profile shows that the Hilbert transform can effectively increase the resolution of the signal, improve the interpretation accuracy of the GPR, and also has a good simulation effect on the complex model.
\end{abstract}

Keywords-GPR, Hilbert transform, simulation

\section{INTRODUCTION}

$\mathrm{GPR}^{[1,2]}$ is a kind of high frequency electromagnetic method. GPR, Similar Radar sounding, uses transmitting antenna with broadband short pulse form to the underground launch of high frequency electromagnetic wave. Then the electromagnetic wave is reflected at the interface of different electromagnetic medium, and received by the receiving antenna. We can achieve the purpose of detecting the space position, scale and physical properties of the underground object by analyzing the characteristics of electromagnetic wave propagation in time and space. Currently, radar interpretation is mainly based on the kinematic characteristics of radar wave, so the study of the dynamic characteristics of radar wave propagation in the underground medium is more conducive to understanding the process of radar wave attenuation. Many scholars use Hilbert for radar signal interpretation ${ }^{[3,4]}$ shows that: multi parameter, dynamic characteristics of real signals calculated by transient spectral analysis $^{[5]}$, can interpret radar profile ${ }^{[6]}$. Classsen et al, on the basis of signal analysis theory, systematically researched the transient spectral theory in 1980. At present transient spectrum theory has been applied in the processing of geophysical data, and it has been very successful ${ }^{[7]}$.

The conventional data processing method of GPR is easy to be affected by the factors such as the clutter interference and the wave mixing. It can leads to poor application effect.
Essentially, Hilbert transform is a kind of the phase lag of the allpass filter, skillfully useing the sine (cosine) relationship ${ }^{[8]}$ of the real part and the imaginary part of analytical expressions. It is also defined instantaneous amplitude, instantaneous phase, and instantaneous frequency at any moment, so we can obtain the instantaneous parameters extraction from complex signal and more abundant information acquisition, compare and analyze the distribution of underground medium. In this paper, Hilbert transform will be used to the forward modeling of GPR data to extract "three instantaneous" information(the instantaneous amplitude, instantaneous phase and instantaneous frequency), which provides a good basis for the subsequent interpretation of radar data.

\section{Method Principle of Hilbert Transform}

\section{A. Basic Concepts of Complex Signal}

According to Fourier transform theory, for any real continuous spectrum signal, the relation $X(t)$ (Real signal) and $X(f)$ (Frequency spectrum) is.

$$
x(t)=\int_{-\infty}^{+\infty} X(f) e^{i 2 \pi t} d f=\int_{0}^{+\infty} X(f) e^{-i 2 \pi f t} d f+\int_{0}^{+\infty} X(-f) e^{-i 2 \pi f t} d f
$$

On one hand, If $x(t)$ only have the positive frequency component, we can obtain

$$
x(t)=\operatorname{Re}\left\{\int_{0}^{\infty} 2 X(f) e^{i 2 \pi f} d f\right\}
$$

On the other hand, $q(t)$ be defined as complex signals of $x(t)$, we can gain

$$
\left\{\begin{array}{l}
q(t)=\int_{0}^{\infty} 2 X(f) e^{i 2 \pi f} d f \\
Q(t)= \begin{cases}2 X(f) & f>0 \\
0 & f<0\end{cases}
\end{array}\right.
$$

\section{B. Hilbert Transform}

When the signal is continuous, we can get formula from forth expressions 


$$
\left\{\begin{array}{l}
q(f)=H^{\prime}(f) X(f) \\
h^{\prime}(t)=\delta(t)+i / \pi t
\end{array}\right.
$$

For $q(t)=h^{\prime}(t) \bullet x(t)=X(t)+i 1 / \pi t \bullet X(t)$, defined $X(t)=1 / \pi t \bullet x(t)$, calling $X(t)$ is $X(t)$ of Hilbert transform, so getting the Hilbert transform factor is $h(t)=1 / \pi t$.

According definition and setting Hilbert transform of $x(t)$ is

$$
R(t)=x(t) \cdot \frac{1}{\pi t}
$$

Instantaneous amplitude, instantaneous phase and instantaneous frequency can be received.

$$
\left\{\begin{array}{l}
A(t)=|q(t)|=\sqrt{x^{2}(t)+R^{2}(t)} \\
\theta(t)=\operatorname{arctg}[x(t)] \\
\omega(t)=[d \theta(t) / d(t)]=d\{\operatorname{arctg}[R(t)] / x(t)\} / d t
\end{array}\right.
$$

\section{CASE ANALYSIS}

The size of the geoelectric model of highway simulation is $60 \mathrm{~cm} \times 10 \mathrm{~cm} \times 30 \mathrm{~cm}$, Fig. 1 . There are a large number of steel wire mesh and 2 steel tubes, that radius are $0.5 \mathrm{~cm}$, are respectively placed in the deep $5 \mathrm{~cm}$ and $32.5 \mathrm{~cm}$ of the model in $\mathrm{Z}$ direction. And two steel tubes are located at $10 \mathrm{~cm}$ and $16 \mathrm{~cm}$ in $\mathrm{X}$ direction. Our numerical simulation method with finite difference time domain, center frequency of antenna simulation is set to $100 \mathrm{MHZ}$, when the window length is 600 ns, sampling 512 points (simulation). As $\Delta x=\Delta y=\Delta z=\Delta s=0.025 \mathrm{~cm}$ and $\Delta t=\Delta s / c \sqrt{3}$, the number of simulation of the grid space is $2400 \times 2400 x 1200$, using UPML to absorb the outward propagation of electromagnetic waves, Sampling distance is $0.4 \mathrm{~cm}$, the line length of $1 \mathrm{~cm}$. We can get the instantaneous amplitude by Hilbert transform of the radar signal, Its schematic as shown in Fig. 1.
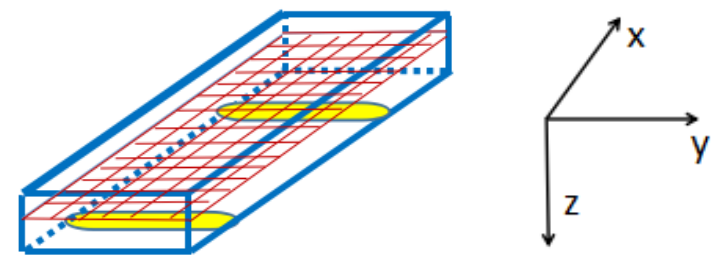

Fig. 1. Schematic diagram of geoelectric model

From the comparison of the results of the 2D profile of three instantaneous information and the original section, the existence of the steel wire mesh and two steel pipes can be seen obviously in Fig. 2. Rectangle represents the position of the steel wire mesh and steel pipe in Fig. A and B. Abnormal reaction more clear than the original section in Fig. A, because the instantaneous amplitude enhanced the medium to radar wave reflection and absorption contrast, in some extent, improving the ability to distinguish the abnormal signal. The arrow indicates the left and right pipe interface in Fig. C. Events more clear than the original section, so this characteristic can be advantageous to the accurate determination of the buried depth of the change of physical property in the inversion interpretation. Instantaneous frequency profile, the two events of arrow being consistent with the position of the steel tube of the instantaneous amplitude diagram, corresponds with instantaneous phase profile very well in fig. D. Its size corresponds to the dominant frequency of the reflected wave. At the same time, it also reflects the two steel tubes around the border. In the inversion process, according to the size and stability of the instantaneous frequency to determine the stability and lithology changes of the underground medium ${ }^{[9]}$.

From the results of $3 \mathrm{D}$ simulation, we find that the simulation results of Hilbert transform can more better reflect the size and depth of the steel wire mesh than the original results in the $Z=5 \mathrm{~cm}$ of $3 \mathrm{D}$ profile of three instantaneous information. Similarly, the two steel tubes of the size and depth can be determined in the $Z=32.5 \mathrm{~cm}$ of three instantaneous information of 3D profile from Fig. 4. However, because of the presence of severe multiple wave interference, he simulation results of the Hilbert transform, compared with the established model, is still not accurate enough.

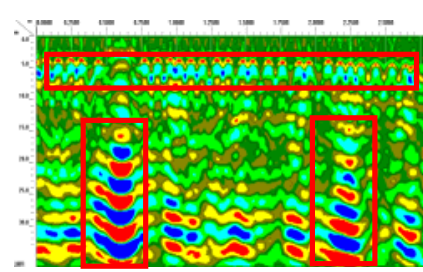

(A) Original section

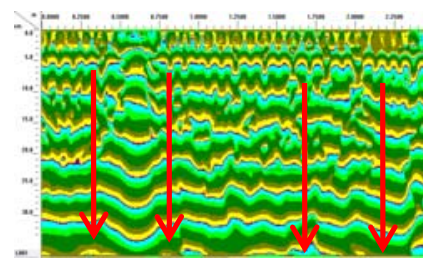

(C) Instantaneous phase profile

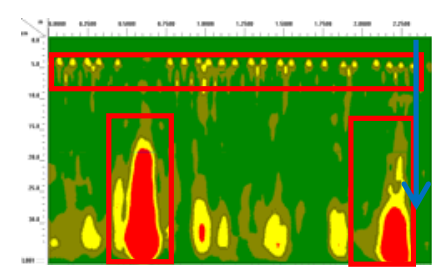

(B) Instantaneous amplitude profile

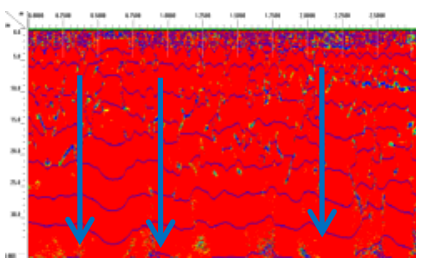

(D) Instantaneous frequency section
Fig. 2. 2D profile of three instantaneous information

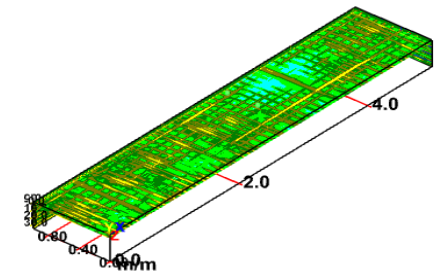

(A) Original section

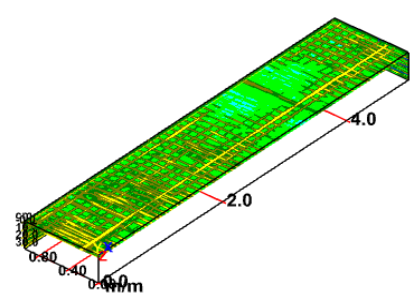

(B)Instantaneous amplitude profile 


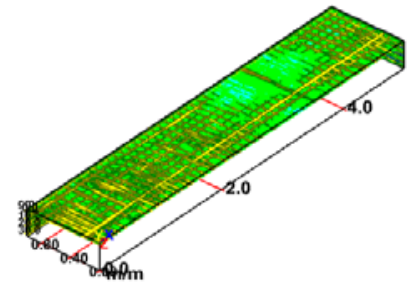

(C) Instantaneous phase profile

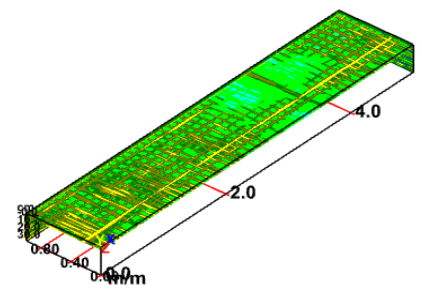

(D) Instantaneous frequency section
Fig. 3. $Z=5 \mathrm{~cm}$ of $3 \mathrm{D}$ profile of three instantaneous information

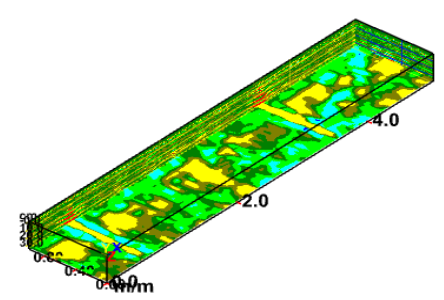

(A) Original section

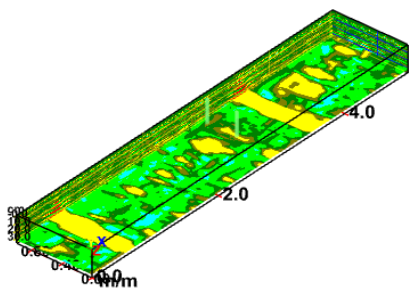

(C) Instantaneous phase profile

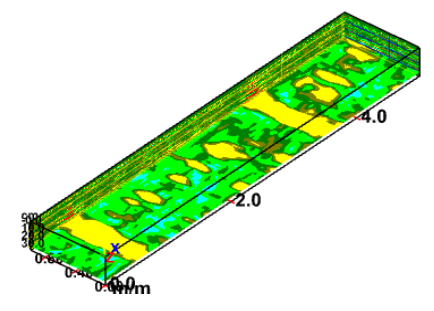

(B) Instantaneous amplitude profile

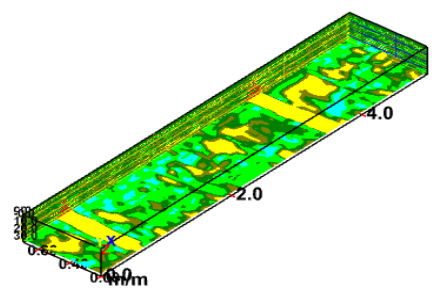

(D) Instantaneous frequency section
Fig. 4. $\mathrm{Z}=32.5 \mathrm{~cm}$ of $3 \mathrm{D}$ profile of three instantaneous information

Furthermore, Because the instantaneous phase and instantaneous frequency are sensitive to the change of the physical properties of the medium ${ }^{[10]}$, so that some parts of the events performance is more disordered, and the effect is not very well.

The instantaneous amplitude section is more clear and accurate in reflecting the relative instantaneous phase and instantaneous frequency of the steel tube from Fig. 3 and Fig. 4.

\section{CONCLUSIONS}

Hilbert transform reveals an equivalent transformation between time domain and frequency domain by Fourier transform. Using the multi-parameters wave profile to comprehensive analysis can avoid the deviation caused by the single radar waveform time range profile, thus improving the accuracy of the interpretation of GPR.

Hilbert transform, as a signal processing algorithm, can effectively extract "three instantaneous" information of GPR from the complex signal. It can be seen from the above analysis and application results that the instantaneous amplitude profile compared with the original results can reflect the complex structure more clearly and accurately; Instantaneous phase and instantaneous frequency can better reflect the fluctuation of the physical interface of the medium, and more accurate judgment of the area of the medium. But the instantaneous phase and instantaneous frequency are sensitive to the change of the physical properties of the medium, so that some parts of the events performance is more disordered.

Hilbert transform has a good response to complex structure. In the process of Hilbert transform, we can get the instantaneous amplitude, instantaneous phase and instantaneous frequency section to strengthen the reflection and absorption contrast of the radar wave.I although the interference of multi-parameters wave, it can improve the resolution of abnormal signals in a certain extent, and help to improve image resolution and target detection of GPR.

\section{REFERENCES}

[1] Dai Q W, Yang H J, et al. 2007. Analysis and application of GPR data. West-China Exploration Engineering(in Chinese), 03:130-131.

[2] Zhang Y D, et al. 2004. Application of Hilbert transform in GPR data. Journal of Engineering Geophysics(in Chinese), 04:349-352..

[3] Yao J. 2007. Study on Application of complex signal analysis technology in GPR data processing. Jilin University..

[4] Cao Z F, et al. 1996. GPR data processing method and its application Geology and Exploration(in Chinese), 01:34-42..

[5] Zhang M, et al. 2003. Study on the instantaneous characteristic parameters of signal using Hilbert transform. Telecommunication Technology(in Chinese), 04:44-48..

[6] Xiao B, He J S. 1999. Transient spectrum analysis of GPR. Geophysical and Geochemical Exploration(in Chinese), 06:459-461+466.

[7] Zheng Zhizhen. 1992. Progress in geophysical signal processing and future directions. Geophysical Progress (in Chinese), 02:36-44.

[8] Yu Z X, et al. 2005. Complex signal analysis technology and in the digital processing of geological radar application. Rock mechanics and Engineering Journal (in Chinese), 05:798-802.

[9] Zhai B, Yang F. 2007. Application of Hilbert transform in GPR data processing. Computer and Information Technology (in Chinese), 08:2930.

[10] Xie X Y, Wan M H. 2000. Complex signal analysis technique in GPR signal processing application. Calculation of Geophysical and Geochemical Exploration Technology (in Chinese), 02:108-11 